\title{
CUCKOO SEARCH-AIDED LMS ALGORITHM FOR CHANNEL ESTIMATION IN MC-CDMA SYSTEMS
}

\author{
${ }^{1}$ Balaji, S. and ${ }^{2}$ N. Vasudevan \\ ${ }^{1}$ Research Scholar, ECE, JNTUH, India \\ ${ }^{2}$ Dean, SRM University, Chennai, India
}

Received 2013-10-04; Revised 2014-01-07; Accepted 2014-01-27

\begin{abstract}
In the progress of transmission systems that uses the diversity in various domains, the execution of competent baseband receivers categorized by affordable computational load is an essential thing. This would be an imperative point in the future expansion of $4 \mathrm{G}$ systems in which the space, time and frequency diversity will be merged together to enhance the system throughput. Here, we develop a channel estimation technique for MC-CDMA system for the minimization of BER and the maximization of throughput. The maximization of throughput is an essential thing for the successful reception of signal. At the receiver side, the original data is obtained based on the channel estimation algorithm and the inverse process of the transmitter side is performed in the receiver side. The major contribution of our work is to estimate the channel information in an adaptive way. We estimate the channel using the cuckoo search algorithm based on the best solution we obtain from the cuckoo search algorithm. After estimating the channel, we calculate the Bit Error Rate (BER) performance and throughput based on the acknowledgement send by the receiver. Experimental results show that our technique is better in terms of BER and throughput compared to the existing technique.
\end{abstract}

Keywords: Cuckoo Search Algorithm, Channel Estimation, MC-CDMA System, Throughput, BER

\section{INTRODUCTION}

As the wireless internet service has been considered as one of the primary services in the future generation wireless systems, competent exploitation of radio resources on downlink is becoming more essential (Klemm et al., 2009). Multi-carrier system has been inspired by huge claim on frequency allocation that results in crowded spectrum and huge numbers of users require concurrent access. Prevailing wireless systems may be exploited single frequency, single antenna and pulse for carrier transmission and reception. In case of malfunction, the total system will become non operational due to the issues of such system. A distributed system by means of multicarrier, multi-antenna and coded pulse can offer a more appropriate communication (Sinha and Borty, 2010) and multi-rate Code-Division Multiple Access (CDMA) packet systems, radio resources can be consigned in a code or time-division manner. Some influential multiaccess designs and error control approaches are essential (Vakil and Aghaeinia, 2009) for successful communications in similar wireless transmission channels. Therefore, Multicarrier Code Division Multiple Accesses (MC- CDMA) which is a mixture of CDMA and multicarrier modulation derived from Orthogonal Frequency Division Multiplexing (OFDM) ( Benhamid and Othman, 2009; Karim et al., 2010) has been tempting much interest and is under wide study. Each user's modulated symbol to be transmitted in MC-CDMA is extending over numerous subcarriers by means of an orthogonal dissemination sequence defined in frequency domain.

The data rate, capacity and the performance of the system is attempted to enhance in wireless communication based on the modern studies. An interesting approach that exploits more than one antenna in transmitter ans receiver is spatial diversity. We exploited as the transmit diversity approach for MC-CDMA system (Vakil and Aghaeinia, 2009). In MC-CDMA designs, the transmitter of both multi-tone depended Direct Sequence CDMA (DS-CDMA) and that of orthogonal multicarrier DS-CDMA spreads the serial to parallel transformed data streams by means of specified spreading code in the time domain. In various

Corresponding Author: Balaji, S., Research Scholar, ECE, JNTUH, India 
ways we can get the mixture of multicarrier transmission and CDMA. Consequently, the multiplexing CDMA (Sayadi et al., 2009; Aliesawi et al., 2011) designs are classified in to two: first one is that the frequency domain spreading in MC-CDMA integrates the multicarrier transmission with the frequency domain spreading i.e., the original data stream from the user is with the user specific spreading code in the frequency domain but not in the time domain. In other words, each signal is transmitted concurrently in different subcarriers but multiplied by respective chips of the spreading code for every subcarrier. The second one is that the Time Domain Spreading termed as MC-CDMA scheme scatters the original user data stream in the time domain (Narwal et al., 2012).

In bit loading strategy implemented to MC- CDMA is offered in the single user case. The algorithm has to allocate bits and energy per code and also to estimate the number of codes that increase the throughput per subcarrier. The throughput tumbles with the increase in channel selectivity is more or less insensitive to channel selectivity when each symbol is widen over all the subcarriers accessible; spreading each symbol over all the subcarriers provides the uppermost throughput and also found that the throughput is more or less independent of the information sequence length. In addition, the relationship of MC-CDMA, OFDM and DS-CDMA with RCPT HARQ exposes that, under same settings, the throughput of MC-CDMA is greatest in a frequency selective fading channel (Gacanin et al., 2012; Ma et al., 2012). Earlier studies on the downlink throughput did not cautiously contemplate the selfinterference and the restricted data rate set. System throughput on the uplink can be enhanced by consenting only one data user to transmit for the reason that the multi-user interference, the self-interference on the synchronous downlink is not much fewer than the multiuser interference, however, not adequate to expose the effects of the self-interference and the restricted data rate set on the system throughput (Chandra and Prabha, 2012; Benhamid and Othman 2009).

In this study, we propose a channel estimation technique for MC-CDMA system for the minimization of bit error rate and the maximization of throughput. The aspect of our technique is inspired by the technique given in (D'Orazio et al., 2011) which they have exploited the genetic algorithm aided LMS algorithm for the channel estimation of MC-CDMA system. Here, we modulate the user data using the Binary Phase Shift Keying (BPSK) technique and we apply the spread spectrum technique on the modulated signal and then apply the inverse fast Fourier transform. We then transmit the inverse fast Fourier transformed signal after applying the cyclic prefix technique to eliminate the inter-symbol interference. At the receiver side, to retrieve the original signal we perform the inverse process that we applied in the transmitter side. After receiving the signal, the receiver will send an acknowledgement indicating whether it received the signal correctly. Using this acknowledgement, we calculate the throughput which is the average rate of successful message delivery in a communication channel. We estimate the channel in MC-CDMA system by using cuckoo search algorithm aided LMS algorithm. This paper is organized as follows: The second section shows the system model of MC-CDMA and the third section explains our adaptive way of estimating channel and the fourth section shows the performance of our proposed technique and the fifth section discusses our technique compared with the existing technique and the sixth section concludes our technique.

\section{SYSTEM MODEL}

The system model for MC-CDMA system is as follows: Initially we set the number of users as $\mathrm{X}=$ $\left\{\mathrm{U}_{1}, \mathrm{U}_{2}, \mathrm{U}_{3}, \ldots, \mathrm{U}_{\mathrm{i}}, \ldots, \mathrm{U}_{\mathrm{m}}\right\}$ where $\mathrm{m}$ denotes the total number of users and $\mathrm{i}$ denotes $\mathrm{i}^{\text {th }}$ user from the set of users $X$. Thereafter, we set the number of data subcarriers $\mathrm{n}$ and the data bit used by each user. The Fig. 1 shows the system model of MCCDMA system. The equation given below shows the data send by the user $i$ :

$$
\mathrm{D}_{\mathrm{U}_{\mathrm{i}}}=\left\{\mathrm{d}_{1}^{\mathrm{U}_{\mathrm{i}}}, \mathrm{d}_{2}^{\mathrm{U}_{\mathrm{i}}}, \mathrm{d}_{3}^{\mathrm{U}_{\mathrm{i}}}, \ldots, \mathrm{d}_{\mathrm{q}}^{\mathrm{U}_{\mathrm{i}}}, \ldots ., \mathrm{d}_{\mathrm{k}}^{\mathrm{U}_{\mathrm{i}}}\right\}
$$

Where:

$$
\begin{aligned}
& \mathrm{D}_{\mathrm{ui}}=\text { Data of } \mathrm{i}^{\text {th }} \text { user } \\
& \mathrm{D}=\text { Data bits } \\
& \mathrm{q}=\mathrm{q}^{\text {th }} \text { data bit } \\
& \mathrm{k}=\text { Total number of data bits }
\end{aligned}
$$

We then generate the walsh code WC based on the data sub-carriers we set. The walsh code is an approach exploited in the wireless communication to transmit multiple replica of data stream across different antennas and to use various received versions of data to enhance the trustworthiness of data transfer. If we set the number of data sub-carriers as $n$, we will get the walsh code in $\mathrm{n} \times \mathrm{n}$ matrix format. A sample format of walsh code $\mathrm{WC}$ for the data sub-carrier $\mathrm{n}$ is as follows:

$$
\mathrm{WC}(\mathrm{n})=\left[\begin{array}{ccccc}
\alpha_{11} & \alpha_{12} & . & . & \alpha_{1 \mathrm{n}} \\
\alpha_{21} & \alpha_{22} & . & . & \alpha_{2 \mathrm{n}} \\
\cdot & . & . & . & . \\
. & . & . & . & \cdot \\
\alpha_{\mathrm{n} 1} & \alpha_{2 \mathrm{n}} & . & . & \alpha_{\mathrm{nm}}
\end{array}\right]
$$


Balaji, S. and N. Vasudevan / Journal of Computer Science 10 (6): 935-947, 2014

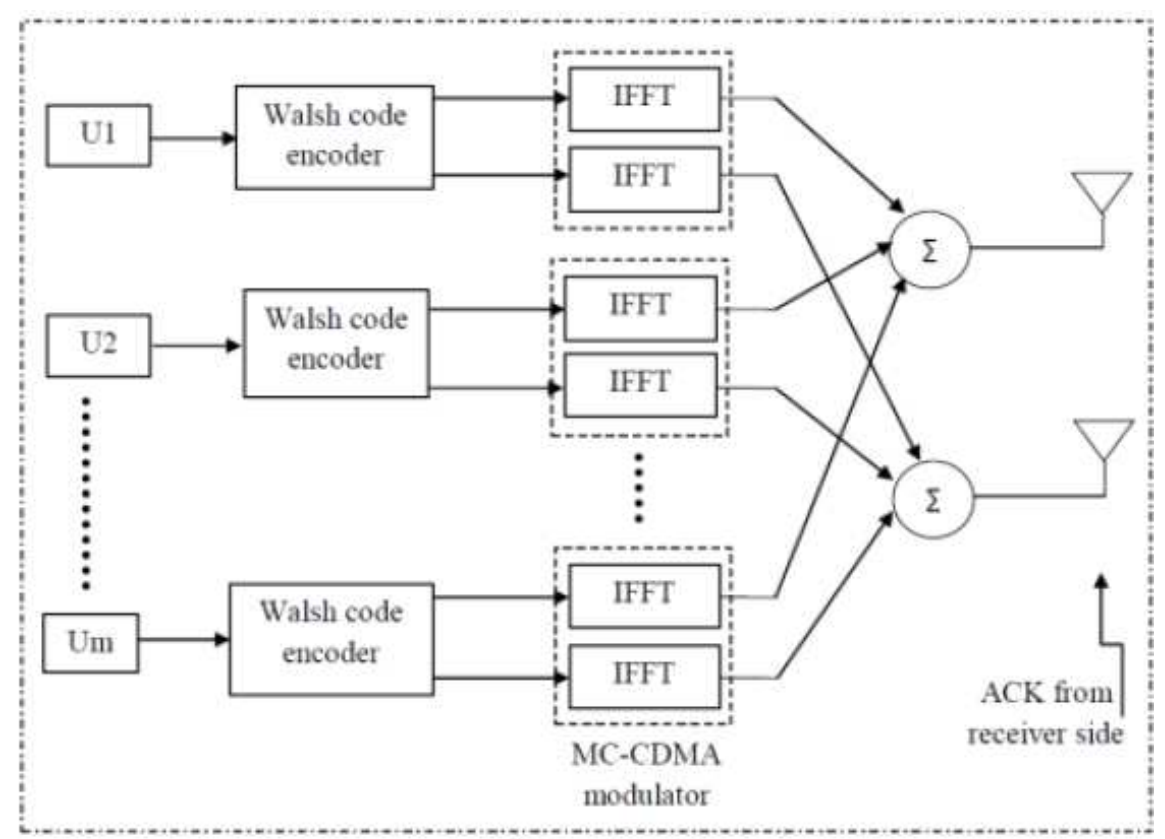

Transmitter side

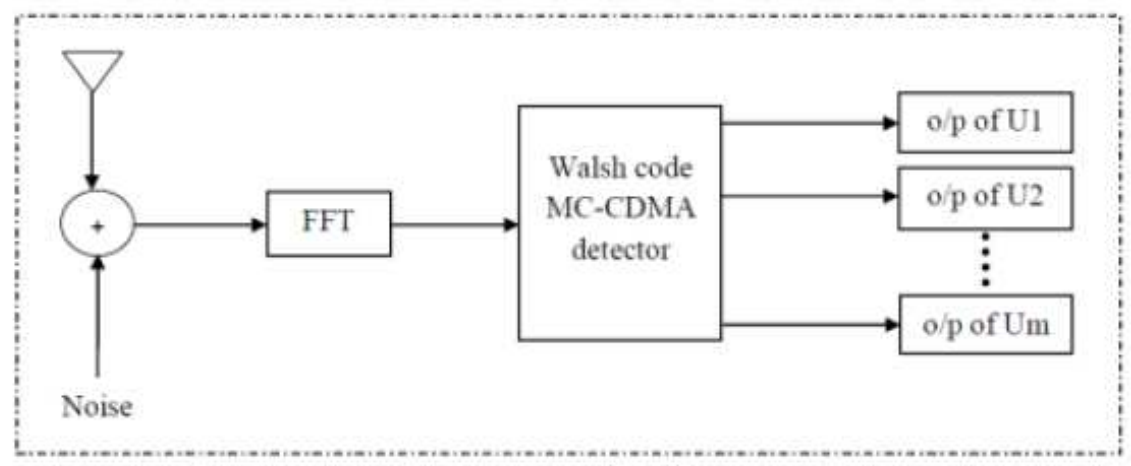

Receiver side

Fig. 1. System model of MC-CDMA system

From this walsh code, we separate the walsh code for the number of users. For instance, the walsh code for the first user, second user and last user are as follows:

Where:

$$
\begin{aligned}
\mathrm{WC}_{\mathrm{U}_{1}} & =\left[\begin{array}{lllll}
\alpha_{11} & \alpha_{12} & . & . & \alpha_{1 \mathrm{n}}
\end{array}\right] \\
\mathrm{WC}_{\mathrm{U}_{2}} & =\left[\begin{array}{lllll}
\alpha_{21} & \alpha_{22} & . & . & \alpha_{2 \mathrm{n}}
\end{array}\right] \\
\mathrm{WC}_{\mathrm{U}_{\mathrm{m}}} & =\left[\begin{array}{lllll}
\alpha_{\mathrm{n} 1} & \alpha_{\mathrm{n} 2} & . & . & \alpha_{\mathrm{nn}}
\end{array}\right]
\end{aligned}
$$

$\mathrm{WC}_{\mathrm{U} 1}=$ Walsh code for first user

$\mathrm{WC}_{\mathrm{U} 2}=$ Walsh code for second user

$\mathrm{WC}_{\mathrm{Um}}=$ Walsh code for $\mathrm{m}^{\text {th }}$ user
The user data is modulated using Binary Phase Shift Keying (BPSK) modulation technique. The modulated signal is as follows:

$$
D_{U_{i}}^{B P S K}(t)=D_{U_{i}}(t) * \sqrt{\frac{2 E_{b}}{T_{b}}} \cos 2 \pi f_{c} t
$$

Where:

$$
\begin{array}{ll}
\mathrm{D}_{\mathrm{U}_{\mathrm{i}}}^{\text {BPS }}(\mathrm{t}) & =\text { BPSK modulated } i^{\text {th }} \text { user's data } \\
\mathrm{f}_{\mathrm{c}} & =\text { Carrier frequency }
\end{array}
$$

The spread spectrum technique is applied for the BPSK modulated signal to widen the bandwidth of the 
signal. The spread spectrum is the product of the transpose of the BPSK modulated signal and the respective walsh code. It is explained by an equation below:

$$
\mathrm{SP}_{\mathrm{U}_{\mathrm{i}}}=\left[\mathrm{D}_{\mathrm{U}_{\mathrm{i}}}^{\mathrm{BPSK}}(\mathrm{t})\right]^{\mathrm{T}} * \mathrm{WC}_{\mathrm{U}_{\mathrm{i}}}
$$

Where:

$$
\begin{aligned}
& \mathrm{SP}_{\mathrm{U}_{\mathrm{i}}}=\text { Spread spectrum of } \mathrm{i}^{\text {th }} \text { user } \\
& \mathrm{WC}_{\mathrm{U}_{\mathrm{i}}}=\text { Walsh code of } \mathrm{i}^{\text {th }} \text { user }
\end{aligned}
$$

The inverse fast Fourier transform is applied after finding the spread spectrum for the BPSK modulated signal. The following equation shows the IFFT of $i^{\text {th }}$ user:

$$
\mathrm{IF}_{\mathrm{i}}=\frac{1}{\mathrm{~m}} \sum_{\mathrm{p}=0}^{\mathrm{m}-1}\left[\mathrm{SP}_{\mathrm{U}_{\mathrm{p}}}\right]^{\mathrm{T}} \mathrm{e}^{\mathrm{j} \frac{2 \pi}{\mathrm{m}} \mathbb{P}} ; \text { where } l \in\{0,1,2, \ldots, \mathrm{m}-1\}
$$

Where:

$\mathrm{IF}_{\mathrm{i}}=$ IFFT of $\mathrm{i}^{\text {th }}$ user

$\mathrm{m}=$ Total number of users

Before transmit the signal, we have to apply the cyclic prefix and after summing the cyclic prefixed signal of every user, we transmit the signal. It is expressed by an equation below:

$$
\mathrm{X}_{\mathrm{T}}=\sum_{\mathrm{i}=1}^{\mathrm{m}} 1 \mathrm{~F}_{\mathrm{i}}
$$

Where:

$\mathrm{X}_{\mathrm{T}}=$ Signal to transmit $\mathrm{T} \mathrm{X}$

The received signal contains the original transmitted signal multiplied with the Rayleigh fading and added with noise. It is shown by an equation below:

$$
\mathrm{X}_{\mathrm{R}}=\mathrm{X}_{\mathrm{T}} \mathrm{H}+\mathrm{n}
$$

Where:

$\mathrm{X}_{\mathrm{R}}=$ Received signal

$\mathrm{H}=$ Rayleigh fading channel

$\mathrm{n} \quad=$ Additive white gaussian noise

To estimate the channel in MC-CDMA system, we exploit the cuckoo search aided LMS algorithm in our proposed technique.

\section{PROPOSED CUCKOO SEARCH AIDED LMS ALGORITHM FOR CHANNEL ESTIMATION}

This section explains our proposed technique to estimate the channel in MC-CDMA system. The received signal contains the Rayleigh fading multiplied with the original transmitted signal and it is added with additive white Gaussian noise. We estimate the Rayleigh channel based on the cuckoo search algorithm. The fast Fourier transform is performed on the received signal after discarding the cyclic prefix and the equation is given below:

$$
\mathrm{FF}_{\mathrm{i}}=\sum_{\mathrm{l}=0}^{\mathrm{m}-1} \mathrm{IFe}^{-\mathrm{j} \frac{2 \pi}{\mathrm{m}} \mathrm{Ip}} ; \text { where } \mathrm{p} \in\{0,1,2, \ldots, \mathrm{m}-1\}
$$

Where:

$\mathrm{FF}=$ Fast Fourier transform

\subsection{Cuckoo Search Algorithm}

The fast Fourier transformed signal is then applies to the cuckoo search algorithm to estimate the channel in MC-CDMA system. The process of cuckoo search algorithm is as follows: initially we have to generate initial population of $r$ host nests by setting upper limit and lower limit. So the nests would be generated between the upper and lower limit. Each nest contains randomly generated Rayleigh channel. The Rayleigh channel would be in complex form and we have to find the fitness for each nest. After calculating the fitness, we set a condition to separate the best nests and worst nests. The worst nests are performed by Levy flights and check the fitness again. Compare the fitness of the best nests and the Levy flight applied nests. Take the nest with highest fitness and give it to next iteration with the newly generated nests for the condition we set initially. The iteration will be repeated until we get the best nest constantly. The Fig. 2 shows sample initial solution generated randomly.

\subsection{Fitness Calculation}

The fitness calculation for the solutions (nests) generated randomly in our technique is based on the bit error rate. The process of calculating the fitness for our technique is as follows: the conjugate of the Rayleigh channel of each nest is multiplied with the solution obtained after applying fast Fourier transform separately. 


\begin{tabular}{|c|}
\hline$H$ matrix of $P_{1}$ \\
\hline$H$ matrix of $P_{2}$ \\
\hline$H$ matrix of $P_{3}$ \\
\hline$\cdot$ \\
\hline$\cdot$ \\
\hline$\cdot$ \\
\hline$H$ matrix of $P_{r}$ \\
\hline
\end{tabular}

Fig. 2. Sample initial solution contains Rayleigh channel

It is shown by an equation below:

$$
\mathrm{X}_{\mathrm{EQ}_{\mathrm{i}}}=\mathrm{FF}_{\mathrm{i}} * \operatorname{conj}\left(\mathrm{H}^{*}\right)
$$

Where:

$\mathrm{X}_{\mathrm{EQi}}=$ Equalized value

$\mathrm{FF}_{\mathrm{i}}=$ Solution obtained after fast fourier transform

$\mathrm{H}^{*} \quad=$ Rayleigh channel of each nest

The solution is then multiplied with the walsh code of the respective user and we take the real values alone and convert it to 1's and 0's based on the condition we set:

$$
\mathrm{X}_{\mathrm{R}_{\mathrm{i}}}=\mathrm{X}_{\mathrm{EQi}} * \mathrm{WC}_{\mathrm{U}_{\mathrm{i}}}
$$

Where:

$\mathrm{X}_{\mathrm{Ri}}=$ Real value

$\mathrm{WC}_{\mathrm{Ui}}=$ Walsh code of $\mathrm{i}^{\text {th }}$ user

$$
\mathrm{x}_{\mathrm{OS}_{\mathrm{i}}}=\left\{\begin{array}{c}
1 ; \text { if } \mathrm{XR}_{\mathrm{i}}\left(\mathrm{d}_{\mathrm{q}}\right)>0 \\
0 ; \text { else }
\end{array}\right.
$$

Where:

$$
\mathrm{X}_{\mathrm{OS}_{\mathrm{i}}}=\text { Converted value }
$$

Thereafter, we have to check the bit error rate between the original transmitted signal and the received signal. The bit error rate is calculated based on the following formula:

$$
\mathrm{BER}_{\mathrm{i}}=\frac{\text { no.of incorrect bits }}{\text { total no.of bits }}
$$

For instance, if the transmitted bit sequence 1101011001 is and the received bit sequence as 0100111101, the number of incorrect bits in the received bit sequence is four and the total number of bits is ten. So, here the bit error rate is 0.4 which is forty percentages.

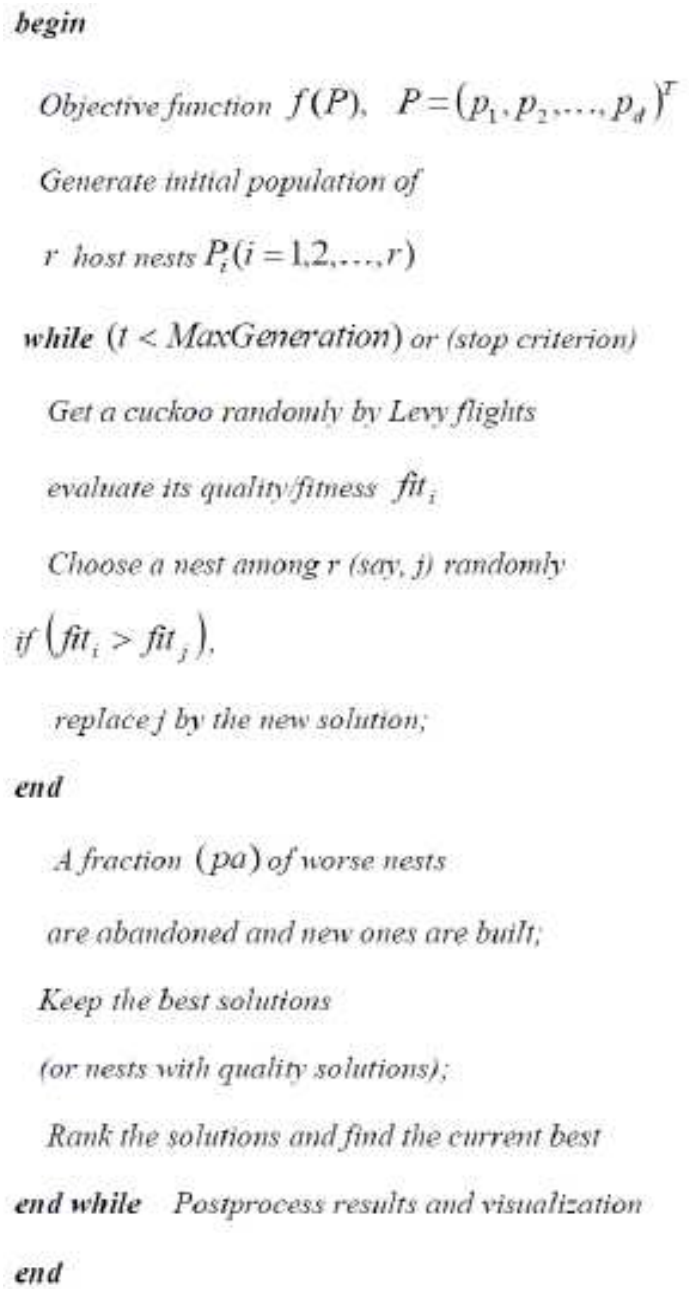

Fig. 3. Pseudo code of cuckoo search algorithm

The equation below shows the formula to calculate the fitness value for each solution (nest) based on the Rayleigh channel in each nest:

$$
\operatorname{fit}\left(\mathrm{P}_{\mathrm{n}}\right)=\sum_{\mathrm{i}=1}^{\mathrm{m}} \mathrm{BER}_{\mathrm{i}}
$$

Where:

$$
\begin{array}{ll}
\text { fit } & =\text { Fitness } \\
\mathrm{P}_{\mathrm{u}} & =\mathrm{u}^{\text {th }} \text { nest ( solution) } \mathrm{u}=1,2, \ldots, \mathrm{r} \\
\mathrm{BER}_{\mathrm{i}} & =\text { Bit error rate of } \mathrm{i}^{\text {th }} \text { user }
\end{array}
$$

The fitness value is calculated for each solution separately using the randomly generated Rayleigh channel. The solutions are then separated based on the best solution and worst solution using the obtained fitness 
value and by setting a threshold limit. In our case, the solution with less bit error rate has the best fitness. The Levy flight function (Yang and Deb, 2009) is applied on the worst solutions and the fitness is calculated again. The Levy flight function is performed as follows:

$$
\mathrm{P}_{\mathrm{u}}^{\mathrm{t}+1}=\mathrm{P}_{\mathrm{u}}^{\mathrm{t}}+\alpha \oplus \operatorname{Levy}(\lambda)
$$

The solutions we obtained after applying Levy flight function and the best solutions are compared and choose the best solution among them based on fitness value. The solution that has best fitness value is given as input for next iteration with the newly generated solutions. The iterations are repeated until we get a constant best solution. The Fig. 3 shows pseudo code of cuckoo search algorithm.

\section{RESULTS}

This section describes the result we obtained for our proposed technique. In this section we compared our proposed technique with the existing technique (D'Orazio et al., 2011) in terms of bit error rate performance and throughput performance. We analyzed both the performance based on number of sub carriers and number of data bits.

\subsection{Evaluation Metrics}

The evaluation metrics we considered are bit error rate and throughput. Both are explained as follows: The bit error rate is the percentage of bits that are received with errors divided by the total number of bits processed over a given period of time. It is shown by an equation below:

$$
\mathrm{BER}=\frac{\text { no.of incorrect bits }}{\text { Total no.of bits }}
$$

Throughput is the average rate of message received successfully in a communication channel. The throughput is typically evaluated in bits per second. If a packet is not received precisely, an outage will occur. This information would be accessible at the receiver through a Cyclic Redundancy Check (CRC) at the Medium Access Control (MAC) layer.

\subsection{Performance Analysis}

The performance of our proposed technique is analyzed using bit error rate and throughput based on number of sub-carriers and number of data bits. In terms of number of sub-carriers, we set the numbers as two, four and eight. In terms of number of data bits, we set the number of data bits as ten thousand and thousand.

\subsection{BER Performance}

\subsubsection{BER Analysis based on Sub-Carriers}

The Fig. 4 show the bit error rate performance based on two sub-carriers. Here, we have measured the bit error rate by varying the signal to noise ratio per bit. In this Fig. 4, we compare the bit error rate of our proposed technique with the bit error rate of the existing technique. From this graph, our proposed technique shows little less bit error rate compared to the existing technique when we set the sub-carriers as two.

The Fig. 5 shows the bit error rate comparison between our proposed technique and the existing technique by setting the sub-carriers as four. This figure also shows that the first user of our technique has the bit error rate little less compared to the existing technique and the bit error rate of the second user of our technique decreases steeply compared to the existing technique by increasing the signal to noise ratio per bit value after $25 \mathrm{db}$. So the overall performance of this graph shows that the bit error rate of our technique is less compared to the existing technique when the sub-carrier is four.

The Fig. 6 shows the bit error rate curve for our proposed technique and the existing technique when the sub-carrier is eight. This graph shows that the first and second user of our proposed technique obtained less bit error rate compared to the existing technique by increasing the value of signal to noise ratio per bit after $15 \mathrm{db}$ when we set the sub-carriers as eight.

\subsection{BER Analysis based on data bits}

The Fig. 7 shows the bit error rate performance of our proposed technique and the existing technique when we set the data bits as ten thousand. Here, the graph shows that the first user of our proposed technique has little less bit error rate compared to the existing technique at some values of signal to noise ratio per bit and it is same to the existing technique at remaining values of signal to noise ratio per bit. Considering the second user of our proposed technique, the bit error rate reduces steeply after $25 \mathrm{db}$ of signal to noise ratio per bit value compared to the existing technique. So overall, our proposed technique has obtained less bit error rate compared to the existing technique.

The Fig. 8 shows the bit error rate comparison of our proposed technique and the existing technique when we set the data bit as thousand. This graph shows that for most of the values of signal to noise ratio per bit, the first and second user of our proposed technique obtained less bit error rate compared to the existing technique. 
Balaji, S. and N. Vasudevan / Journal of Computer Science 10 (6): 935-947, 2014

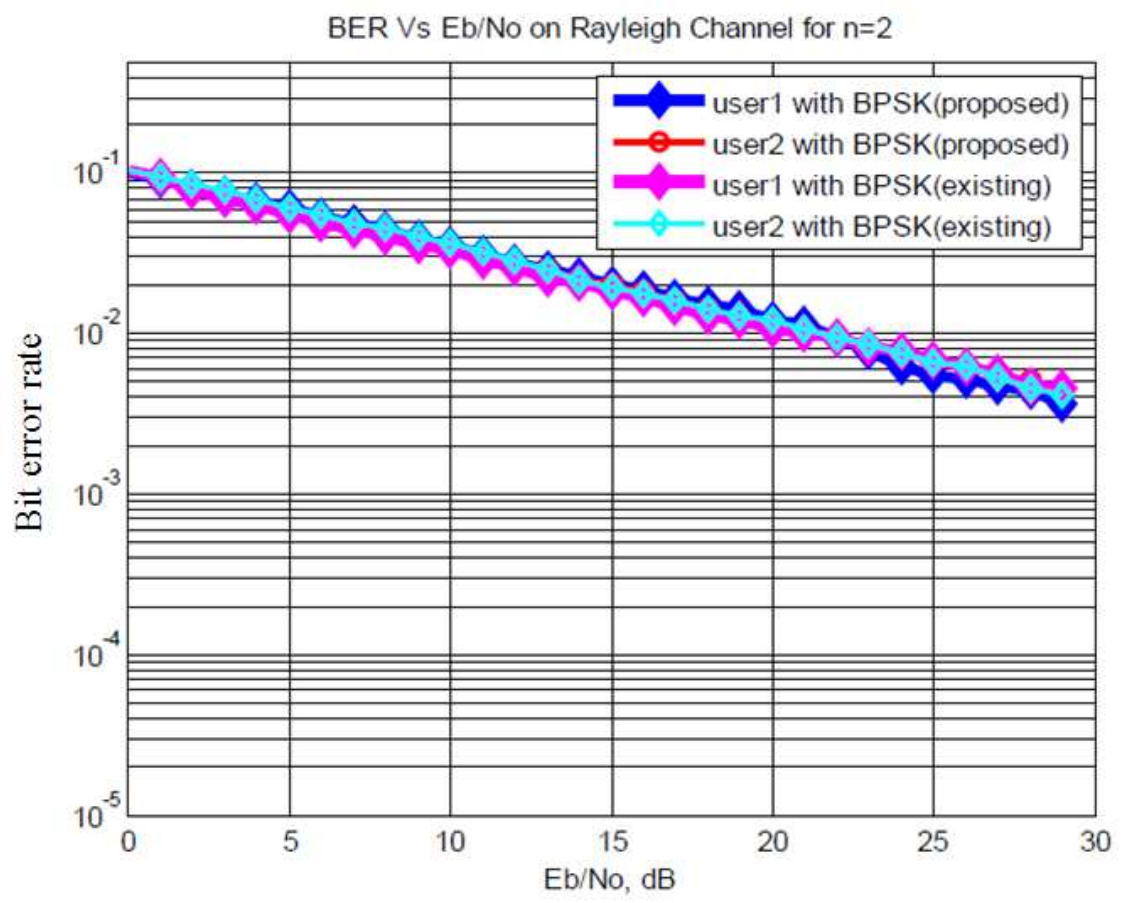

Fig. 4. BER Curve for $n=2$

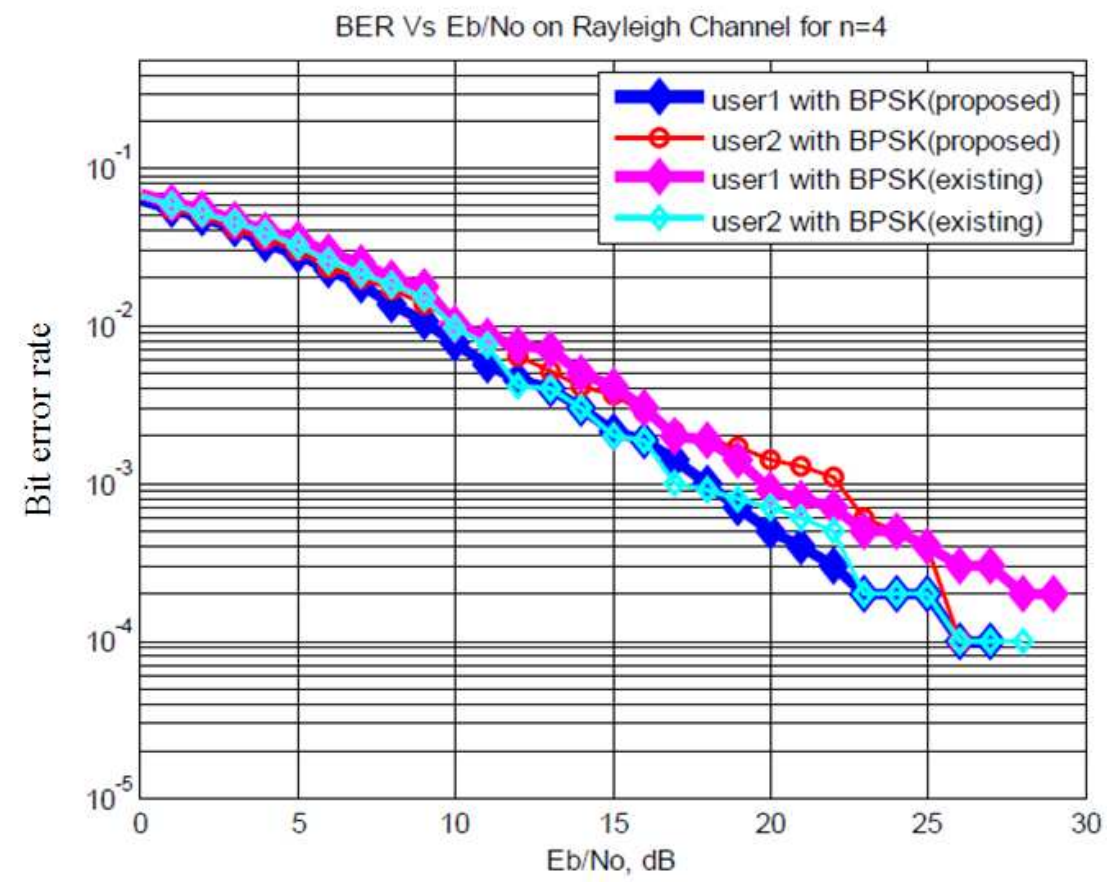

Fig. 5. BER Curve for $n=4$ 
Balaji, S. and N. Vasudevan / Journal of Computer Science 10 (6): 935-947, 2014

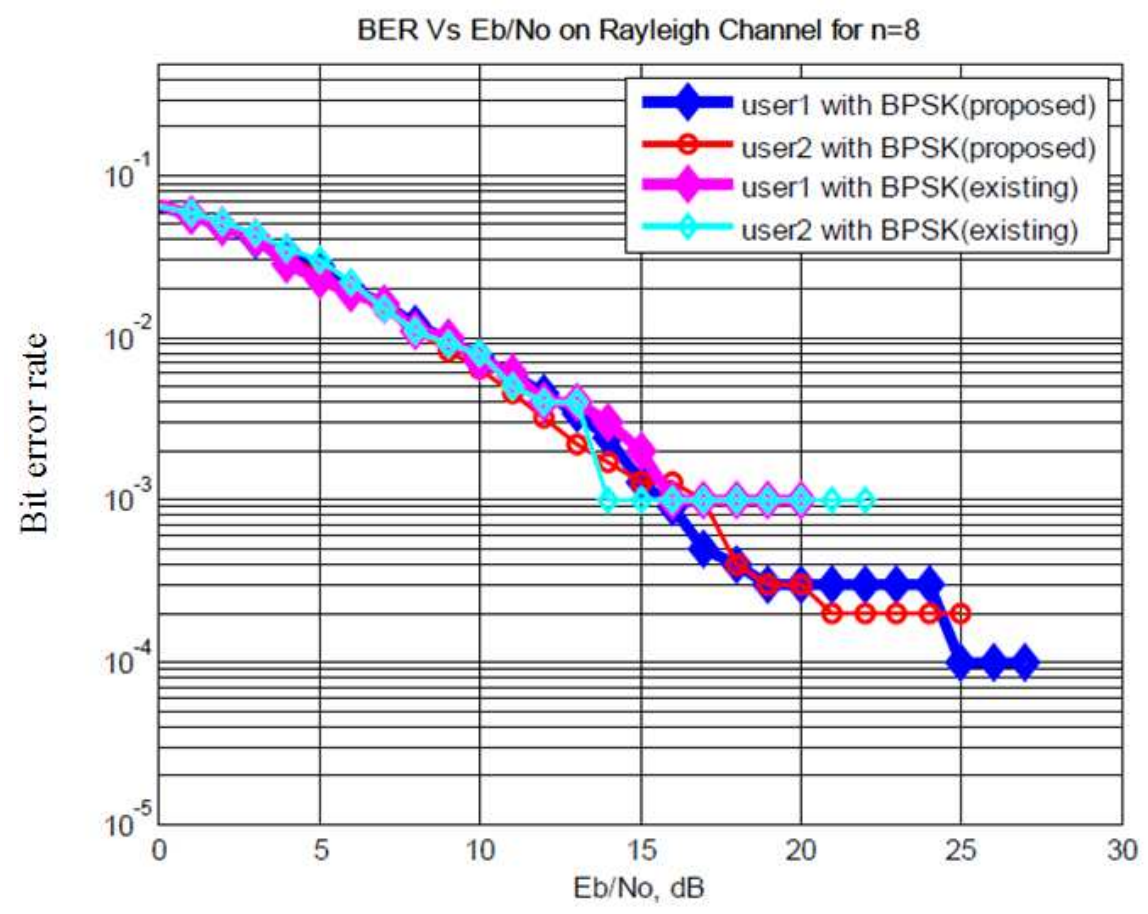

Fig. 6. BER Curve for $n=8$

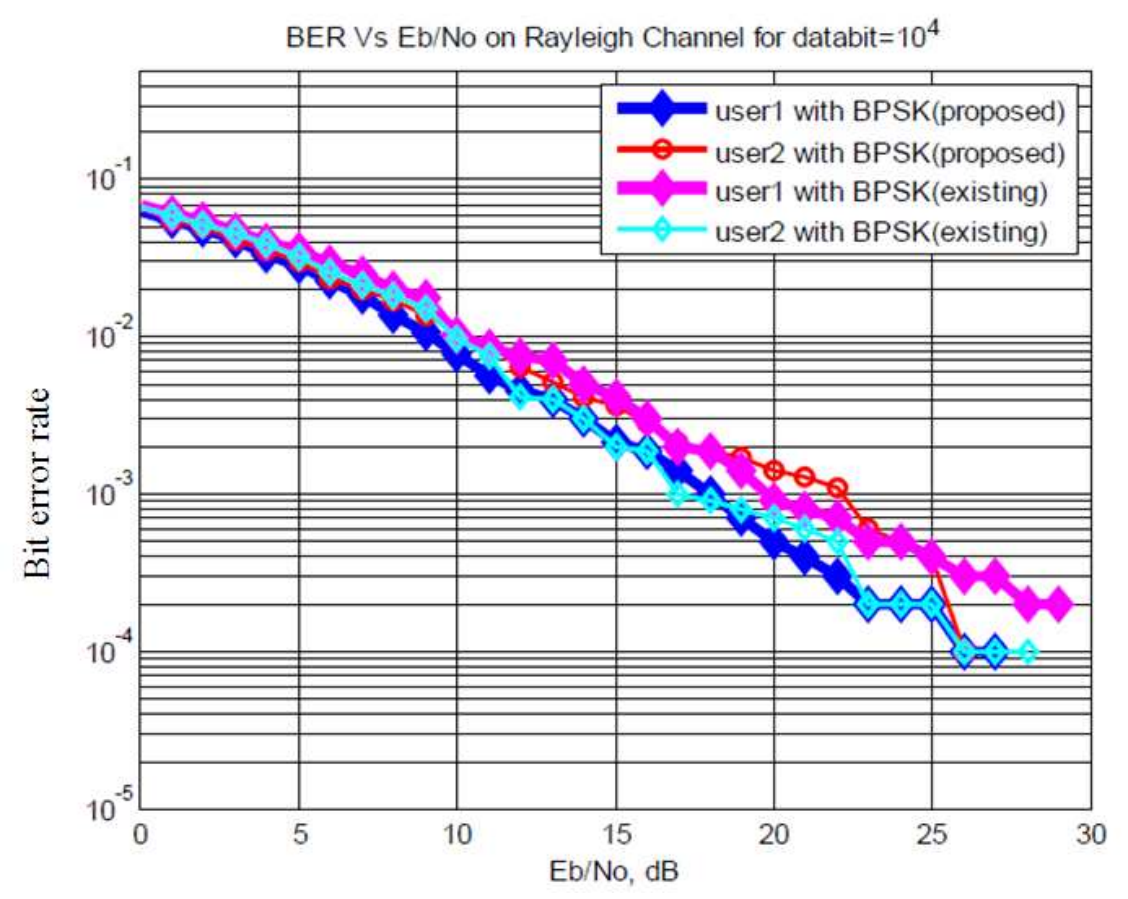

Fig. 7. BER Curve for data bit $=10000$ 
Balaji, S. and N. Vasudevan / Journal of Computer Science 10 (6): 935-947, 2014

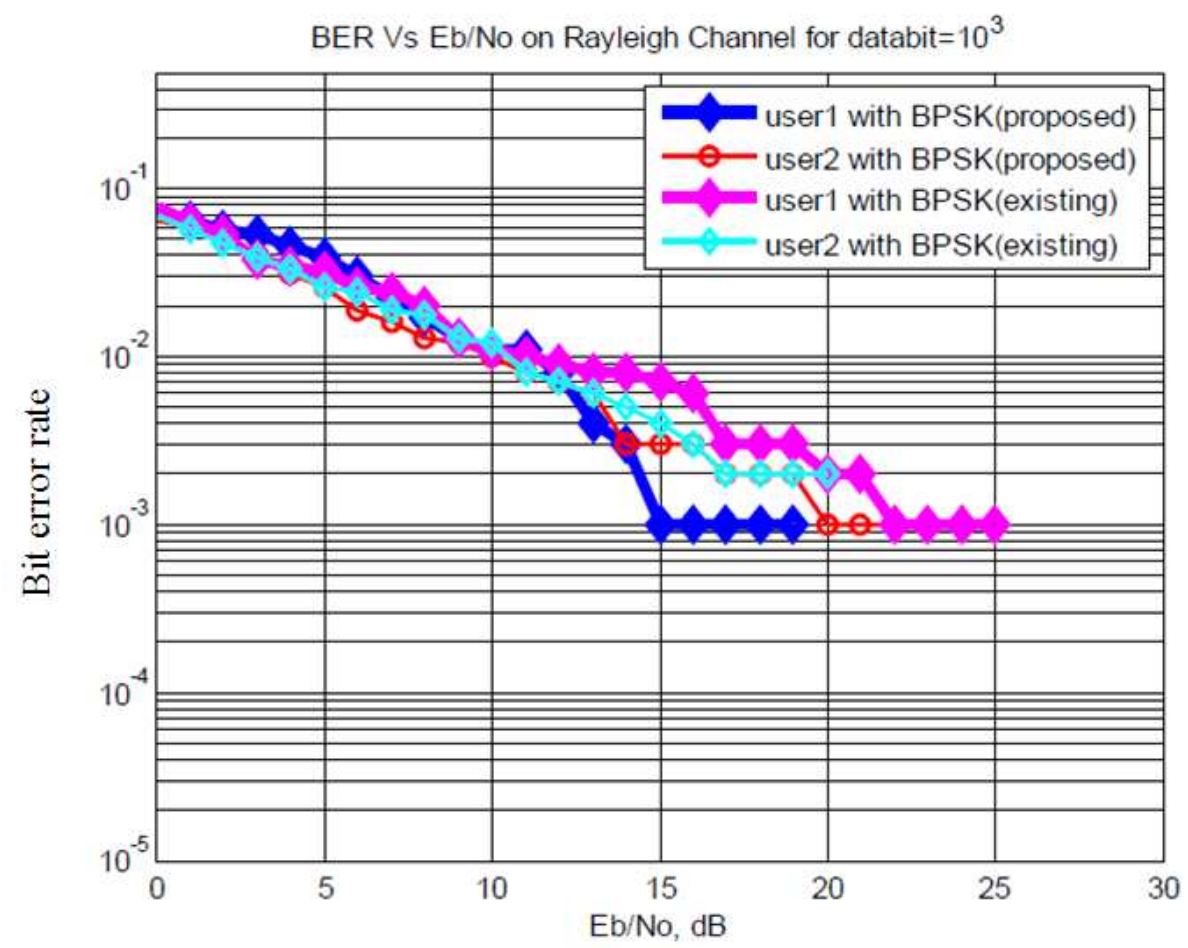

Fig. 8. BER Curve for data bit $=1000$

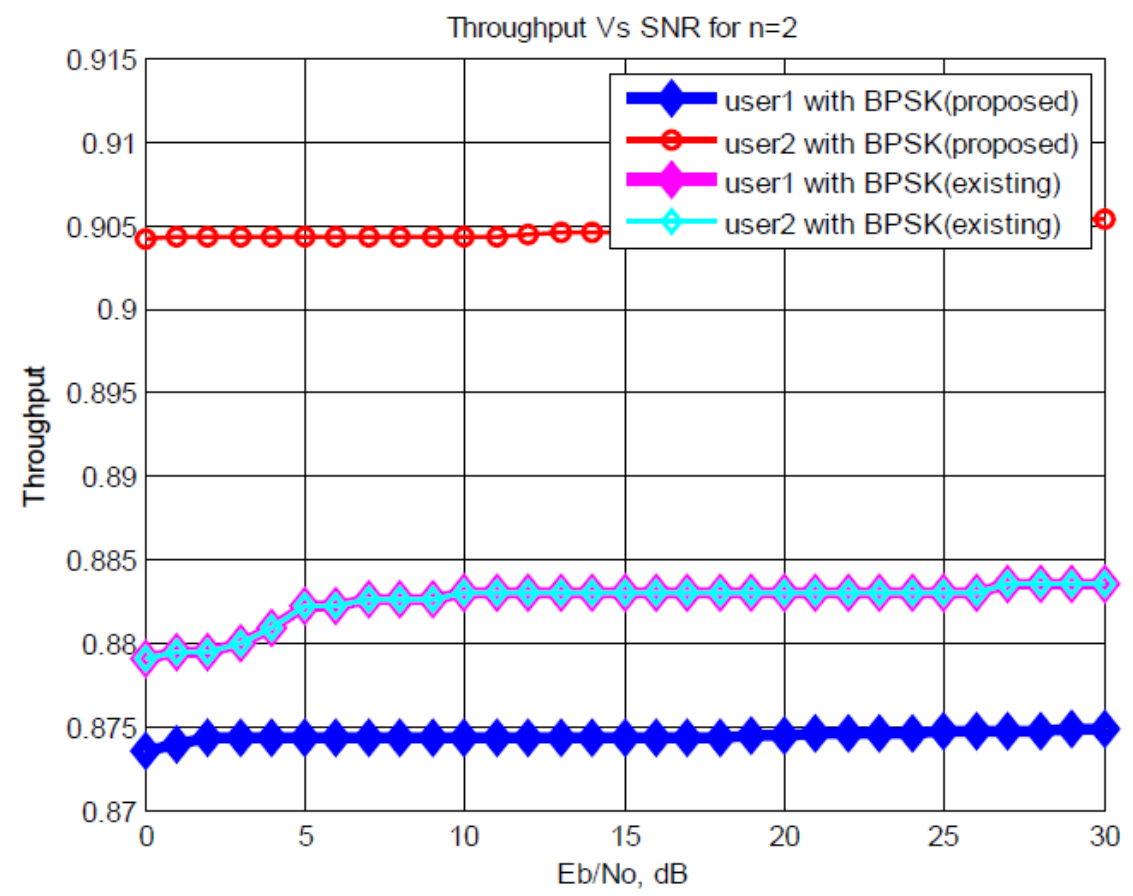

Fig. 9. Throughput curve for $n=2$ 
Balaji, S. and N. Vasudevan / Journal of Computer Science 10 (6): 935-947, 2014

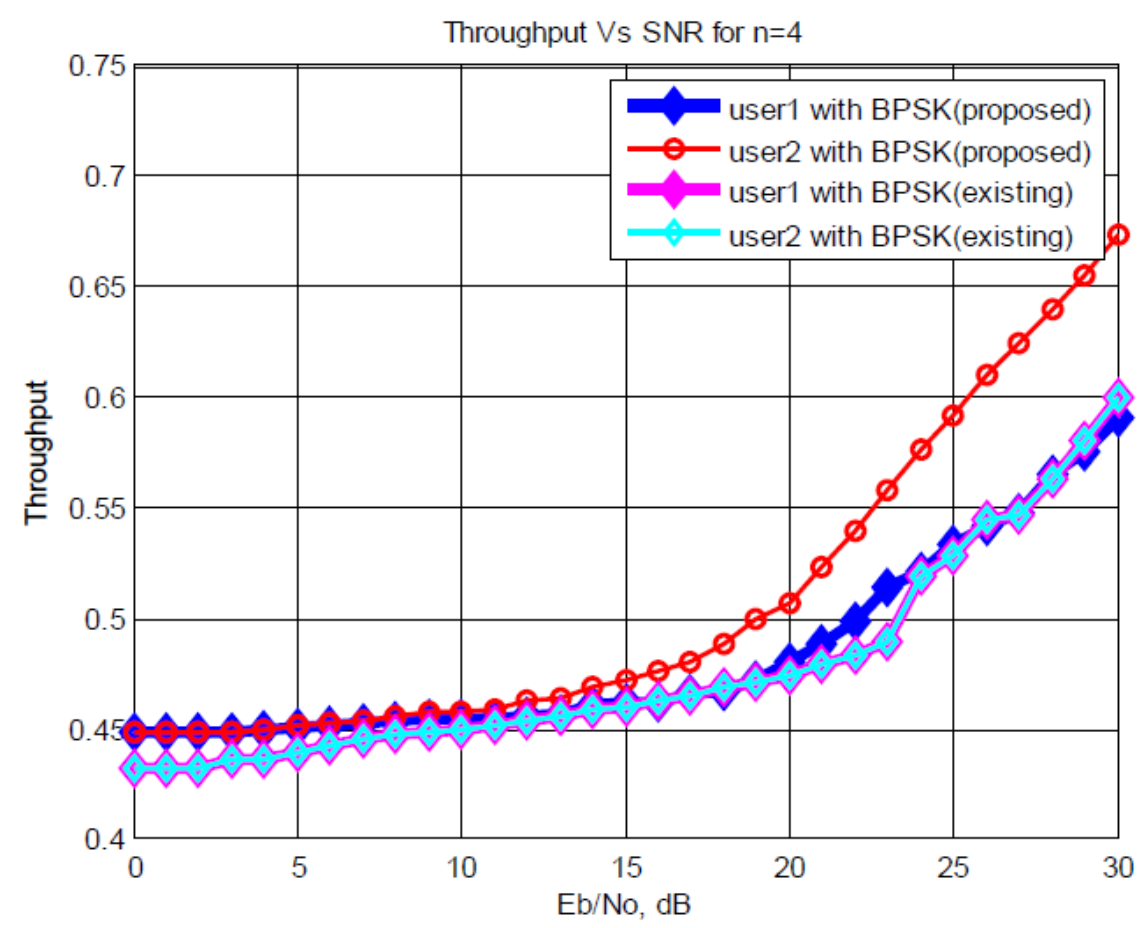

Fig. 10. Throughput curve for $n=4$

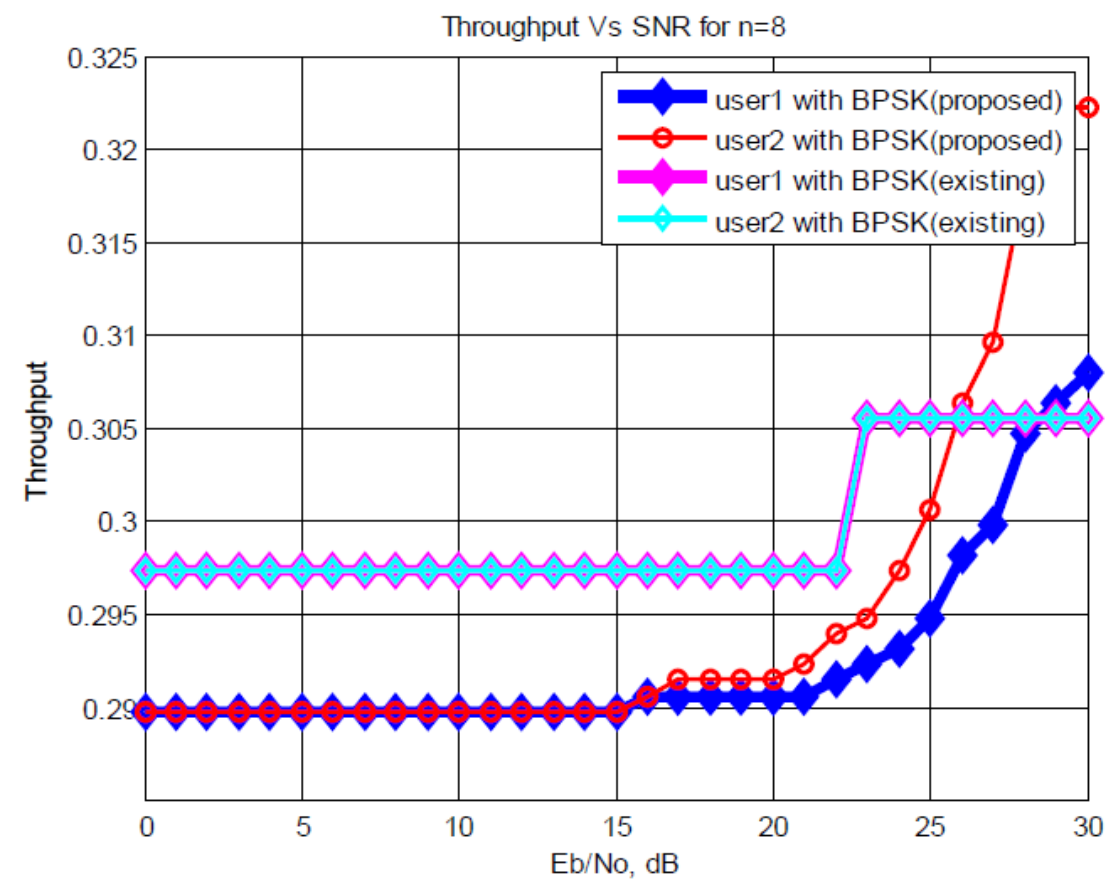

Fig. 11. Throughput Performance when $n=8$ 
Balaji, S. and N. Vasudevan / Journal of Computer Science 10 (6): 935-947, 2014

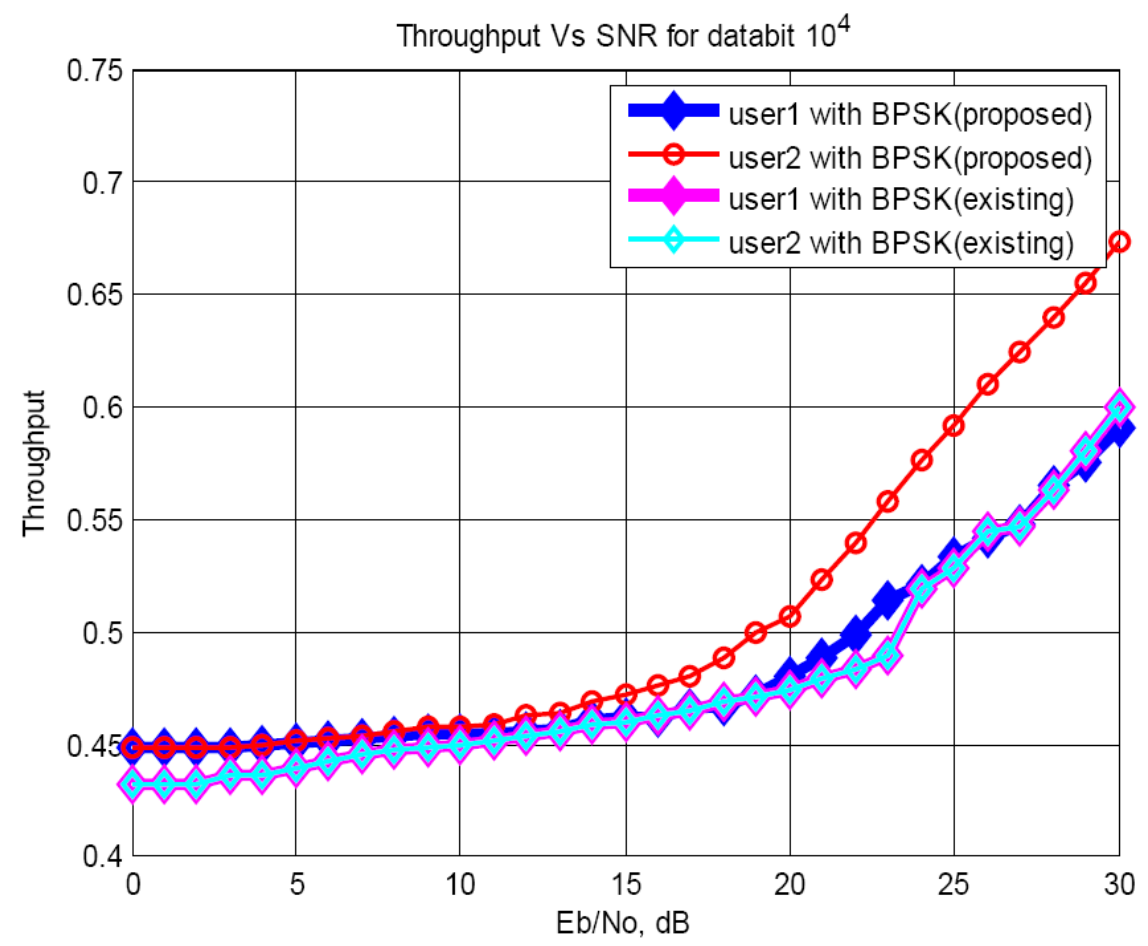

Fig. 12. Throughput curve for data bit $=10000$

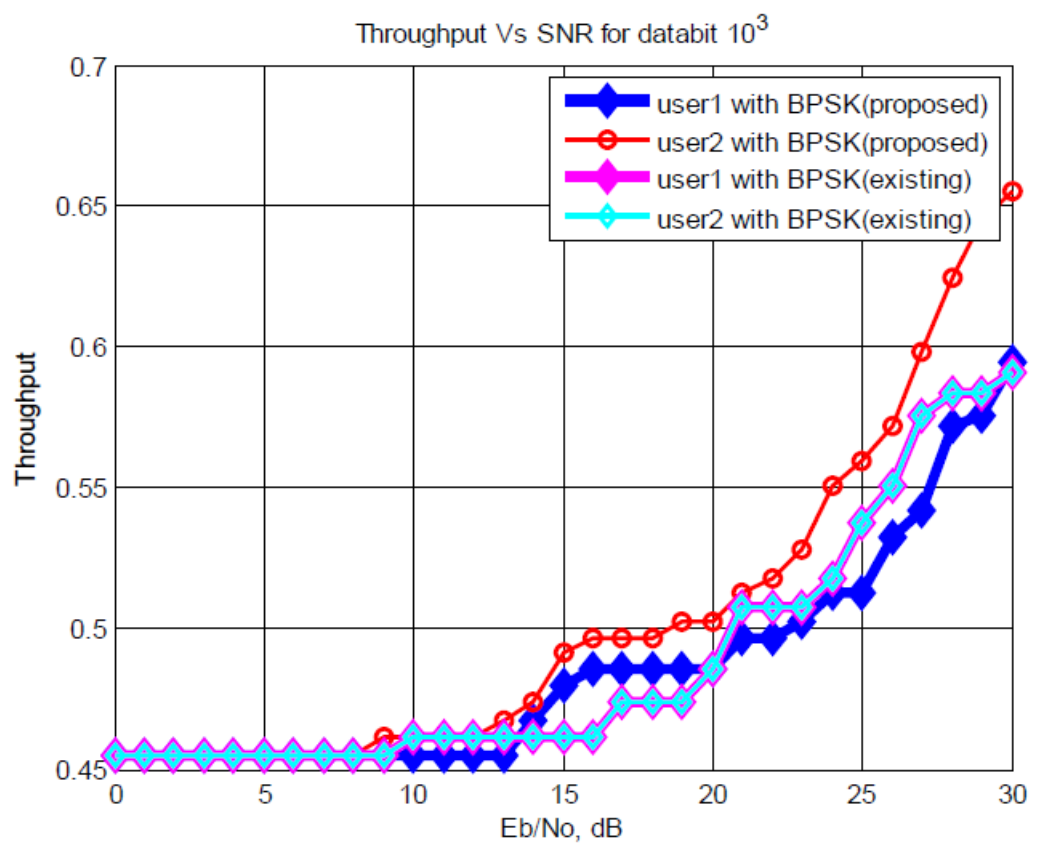

Fig. 13. Throughput comparison when data bit $=1000$ 


\subsection{Throughput Performance}

\subsubsection{Throughput Analysis based on sub- carriers}

The Fig. 9 shows the throughput analysis based on two sub-carriers. The value of throughput is taken by varying the signal to noise ratio per bit. Here, we compare the throughput of our proposed technique with the throughput of the existing technique. This Fig. 9 shows that the throughput of the second user of our proposed technique shows better performance than the users of existing technique.

The Fig. 10 shows the analysis of throughput using four sub-carriers. Here, the first and second users of our proposed technique showed good throughput performance compared to the existing technique when we increasing the value of signal to noise ratio per bit. The Fig. 11 shows the throughput analysis of our proposed and existing technique when the sub-carrier is eight. In this Fig. 12, when we increasing the signal to noise ratio per bit value over $26 \mathrm{db}$, our proposed technique achieved better throughput compared to the existing technique.

\subsection{Throughput Analysis based on data bits}

The Fig. 12 shows the throughput performance of our proposed and existing techniques when the data bit is ten thousand. Here, also the throughput performance of the second user of our proposed technique showed better performance compared to the existing technique and the first user of our proposed technique is little better compared to the existing technique.

The Fig. 13 shows the performance of throughput of our proposed technique and the existing technique when we set the data bit as thousand. Here, the second user of our proposed technique showed better throughput compared to the existing technique; and when comparing the first user of our proposed technique with the existing technique, our technique has better performance in some values of signal to noise ratio per bit.

\section{DISCUSSION}

Our technique is compared with the existing technique (D'Orazio et al., 2011) that used genetic algorithm for channel estimation. We compared the performances in terms of BER based on sub-carriers, BER based on data bits, throughput based on sub-carriers and throughput based on data bits. We set different number of sub-carriers and different value of databits to estimate the performance of our technique with the existing technique. Also, we set the number of users as two for both the techniques. When we compared the performance of BER based on different number subcarriers i.e. setting sub-carriers as two, four and eight, our technique achieved less bit error rate overall and it denotes that our technique performed well than the existing technique that uses genetic algorithm for channel estimation. When we compared the performance of BER based on different data bits i.e. setting data bits as thousand and ten thousand, our technique obtained less bit error rate than the existing one. When we compared the throughput based on different sub-carriers and differnt data bits, the overall performance of our technique performed well than the existing technique. These outcomes implies that for channel estimation, cuckoo search performed better than genetic algorithm.

\section{CONCLUSION}

In this study we have proposed a technique to estimate the channel for MC-CDMA system for minimization of BER and the maximization of throughput. Here, we modulated the user data using BPSK modulation and we applied the spread spectrum technique on the modulated signal and applied the inverse Fourier transform. The cyclic prefix technique is then applied before transmitting the signal from the transmitter side. At the receiver side, the reverse process is performed to retrieve the original signal and the acknowledgement is sent to the transmitter from the receiver side. We estimated the channel information in an adaptive way based on cuckoo search algorithm. We compared the performance of our proposed technique with the existing technique in terms of BER and throughput and showed that our proposed technique is better compared to the existing technique. In future, we can use some hybrid algorithms to estimate the channel information instead of cuckoo search algorithm to enhance the throughput further, because the channel estimation is an important step to enhance the throughput for the successful delivery of message over the communication channel.

\section{REFERENCES}

Aliesawi, S., C.C. Tsimenidis, B.S. Sharif and M. Johnston, 2011. Adaptive channel estimation for multiuser underwater communication systems. Am. J. Eng. Applied Sci., 4: 556-565. DOI: 10.3844/ajeassp.2011.556.565 
Benhamid, M. and M.B. Othman, 2009. Hardware implementation of a genetic algorithm based canonical singed digit multiplierless fast fourier transform processor for multiband orthogonal frequency division multiplexing ultra wideband applications. J. Math. Stat., 5: 241-250. DOI: 10.3844/jmssp.2009.241.250

Chandra and H, Prabha, 2012. Enhancement of quality of service parameters in WiMAX mobile networks. Am. J. Applied Sci., 9: 1906-1915. DOI: 10.3844/ajassp.2012.1906.1915

D'Orazio, L., C. Sacchi, M. Donelli, J. Louveaux and L. Vandendorpe, 2011. A near-optimum multiuser receiver for STBC MC-CDMA systems based on minimum conditional BER criterion and genetic algorithm-assisted channel estimation. EURASIP J. Wireless Commun. Network., 2011: 351494351494. DOI: $10.1155 / 2011 / 351494$

Gacanin, H., M. Salmela and F. Adachi, 2012. Performance analysis of analog network coding with imperfect channel estimation in a frequencyselective fading channel. IEEE Trans. Wireless Commun., 11: 742-750. DOI: 10.1109/TWC.2011.122211.110326

Karim, A.M., M.S. Islam and M. Othman, 2010. Orthogonal frequency division multiplexing timing synchronization in multi-band orthogonal frequency division multiplexing ultra-wideband systems. Am. J. Applied Sci., 7: 420-427. 10.3844/ajassp.2010.420.427

Klemm, L., C. Duy, I. Iacobucci, S. Kuchen and G. Von Levetzow et al., 2009. The B cell mutator AID promotes B lymphoid blast crisis and drug resistance in chronic myeloid leukemia. Cancer Cell, 8: 232245. DOI: 10.1016/j.ccr.2009.07.030, PMID: 19732723

Li, D., Y. Li, H. Zhang and L.J. Cimini, 2012. integer frequency offset estimation for OFDM systems with residual timing offset over frequency selective fading channels. IEEE Trans. Vehicular Technol., 61: 2848-2853. DOI: 10.1109/TVT.2012.2195234
Ma, T.M., Y.S. Shi and Y.G. Wang, 2012. A low complexity MMSE for OFDM systems over frequency-selective fading channels. IEEE Commun. Lett., 16: 304-306. DOI: 10.1109/LCOMM.2012.012412.112328

Narwal, P., S. Singh and S.V.A.V. Prasad, 2012. Performance analysis of BER improvement for multi carrier CDMA system. Int. J. Eng. Innovat. Technol., 2: 72-74.

Ngo, D.T., C. Tellambura and H.H. Nguyen, 2009. Efficient resource allocation for OFDMA multicast systems with spectrum-sharing control. IEEE Trans. Vehicular Technol., 58: 4878-4889. DOI: 10.1109/TVT.2009.2027331

Sayadi, F., M. Ismail, N. Misran, K. Jumari and M. Abdullah, 2009. Efficient detection algorithm for a multiple-input and multiple-output multiuser multicarrier code division multiple access in time-varying channels. Am. J. Eng. Applied Sci., 2: 635-642. DOI: 10.3844/ajeassp.2009.635.642

Sinha, N.B., and S.C. Borty, 2010. Performance evaluation of hybrid-multicarrier access schemes for broadband wireless communication. Int. J. Sci. Technol., 2: 519-526.

Vakil, V. and H. Aghaeinia, 2009. Throughput analysis of STS-based CDMA system with variable spreading factor in non-frequency selective Rayleigh fading channel. Comput. Electr. Eng., 35: 528-535. DOI: 10.1016/j.compeleceng.2008.08.001

Yang, X.S. and S. Deb, 2009. Cuckoo search via lévy flights. Proceedings of the World Congress on Nature and Biologically Inspired Computing, Dec.9-11, IEEE Xplore Press, Coimbatore, pp: 210-214. DOI: 10.1109/NABIC.2009.5393690 